\title{
TEMPERATURE-COMPENSATED TETHERLESS BULK ACOUSTIC PHONON TRAP FOR SELF-OVENIZED OSCILLATORS
}

\author{
R. Tabrizian, A.Norouzpour-Shirazi and F. Ayazi \\ Georgia Institute of Technology, Atlanta, Georgia, USA
}

\begin{abstract}
This paper reports on a novel temperature-compensated single-crystal silicon resonator that has a quadratic temperature characteristic with a high turn-over temperature. An energytrapped resonance mode is synthesized from acoustic coupling of evanescent and propagating phonons with opposite temperature sensitivity trends in a $<100>$-aligned engineered waveguide (phonon trap). A $77 \mathrm{MHz}$ device implemented in AlN-on-silicon platform shows a $Q$ of 13,000 and a turn-over temperature of $87^{\circ} \mathrm{C}$ facilitating implementation of an oven-controlled frequency reference. An oscillator implemented using this device, while selfovenized by a DC current passing through its body, exhibits a consistent phase-noise of $-106 \mathrm{dBc} / \mathrm{Hz}$ at $1 \mathrm{kHz}$ offset from carrier.
\end{abstract}

\section{INTRODUCTION}

For the past decade, MEMS resonators have been promising realization of highly-stable frequency references as an integrated alternative to replace their Quartz-based discrete counterparts. Although MEMS oscillators have been successful in surpassing Quartz references in several performance metrics including phasenoise and long-term stability [1], however large temperature sensitivity of these devices has remained a big challenge, especially as MEMS oscillators are targeting TCXO and OCXO markets with sub-ppm and sub-ppb instability requirements respectively. To address this challenge, a large variety of devicelevel [2,3] and circuit/system-level [4] temperature compensation techniques have been proposed. However, these techniques usually impose large deviation from resonator design and implementation baseline, including excessive power consumption or manufacturing complexities.

In this paper we leverage single-crystal silicon as a designable and controllable thermo-mechanical platform to realize flexible, yet accurate, control on the temperature characteristic of the resonator frequency. Evanescent phonons with highly-positive temperature coefficient of frequency $(T C F)$ are acoustically coupled to propagating phonons with negative $T C F$ to create a temperature-stable resonance mode in a geometrically-engineered acoustic waveguide. Proper distribution of acoustic energy in propagating and evanescent fields in such structure not only provides compensation of the linear $T C F$, but also substantially eliminates support-loss through trapping propagating phonons in the central region of the acoustic cavity and far from anchoring regions (hence the name phonon trap). This, in turn, obviates the need for narrow tethers to anchor the device to the substrate and facilitates self-ovenization of the device by passing a DC current uniformly distributed across the cross-section of the cavity.

\section{TEMPERATURE-COMPENSATED PHONON TRAP}

Proper cascading and geometry engineering of multiple waveguides complying with displacement and strain continuity conditions at transition boundaries facilitate realization of synthesized modes in a phonon trap [5]. While a central waveguide supports propagating phonons, waveguides in flank regions can only support evanescent phonons with exponentially decaying energy as moving towards the substrate. Figure 1 demonstrates the concept of phonon trap through dispersion characteristics of guided waves in rectangular waveguides. The synthesized mode is highlighted with stars on different branches.

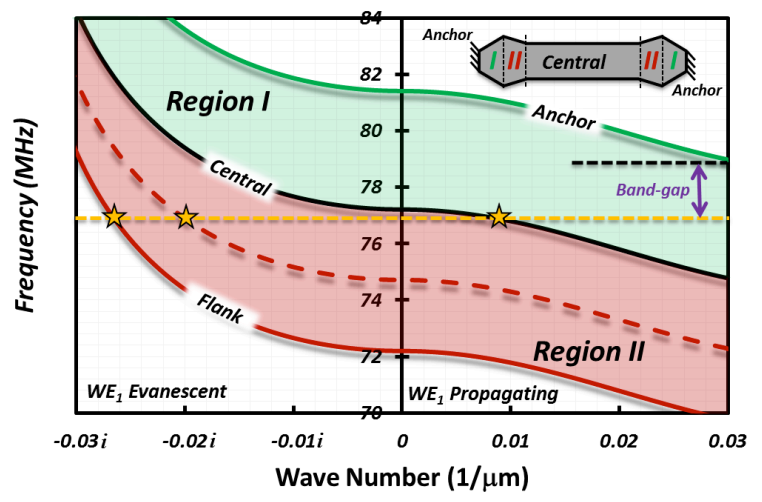

Figure 1: Dispersion characteristic of the first width-extensional $\left(W E_{l}\right)$ phonon branch for different regions of the $<100>$ phonon trap. Proper engineering of flanks and anchors facilitate acoustic energy trapping in the center of cavity and far from substrate.

Since the device is engineered to exclusively trap the energy of a single phonon-type, various spurious modes which are commonly excited in piezoelectrically-transduced resonators are efficiently suppressed as their energy leaks to the substrate through wide anchors of the structure.

Figure 2 shows the TCF behavior of different phonon branches of a single waveguide, exhibiting highly different characteristics for different phonon types, as well as opposite trends in evanescent and propagating portions of each branch.

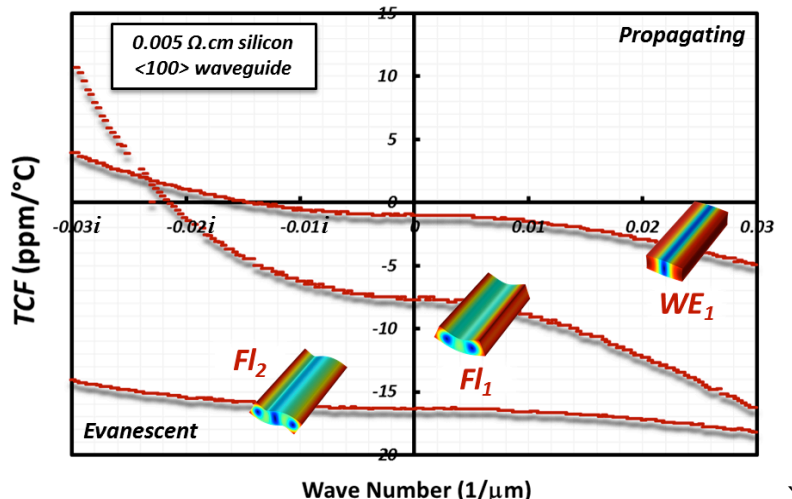

Figure 2: COMSOL-simulated TCF behavior of propagating and evanescent phonons for different branches of a rectangular waveguide aligned in $\langle 100\rangle$ direction of $\mathrm{N}$-doped (100) SOI substrate with a resistivity of $0.005 \Omega . \mathrm{cm}$.

\section{DEVICE CHARACTERIZATION AND DISCUSSION}

Figure 3 shows the SEM image of the $<100>$-aligned phonon trap implemented on the same chip with two conventional silicon bulk acoustic resonators (SiBAR) aligned to $<100>$ and $<110>$ directions to highlight the efficiency of temperature compensation by comparing their temperature behavior.
Solid-State Sensors, Actuators and Microsystems Workshop Hilton Head Island, South Carolina, June 8-12, 2014 


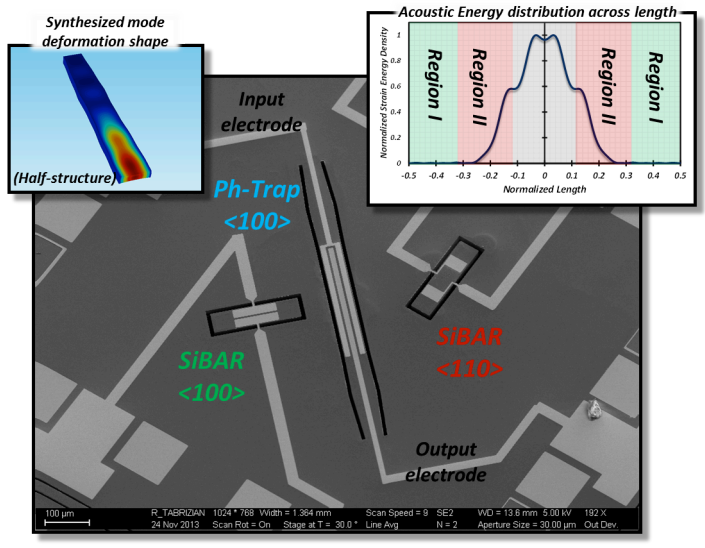

Figure 3: SEM image of $<100>$-aligned phonon trap adjacent to two conventional SiBARs aligned in $<100\rangle$ and $<110\rangle$ crystallographic directions. The insets show the synthesized modeshape and its energy distribution across device length.

Figure 4 shows the large-span frequency response of the phonon trap. Spurious modes are efficiently suppressed as a result of selective energy trapping and tether elimination.

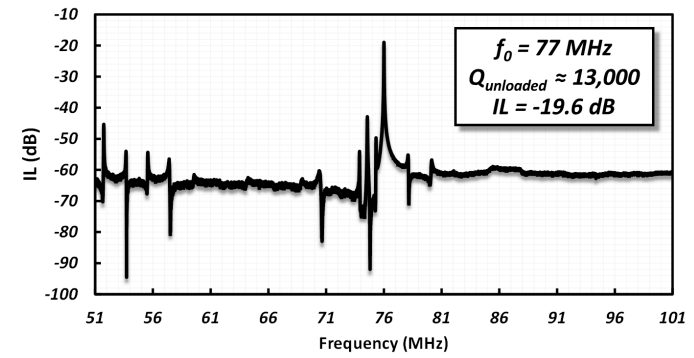

Figure 4: Large-span frequency response of the phonon trap.

Figure 5 compares the temperature characteristic of resonance frequency for the trap and SiBARs of Figure 3, showing the effectiveness of temperature compensation technique.

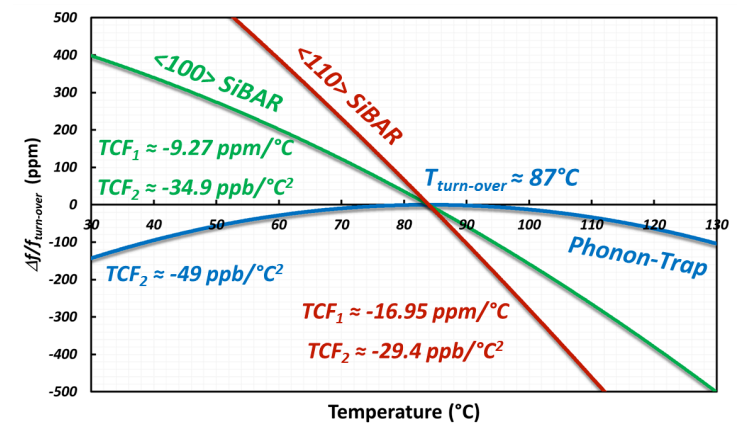

Figure 5: Temperature characteristic of the $<100>$ phonon trap in comparison with conventional SiBARs aligned to $\langle 100\rangle$ and $<110>$ crystallographic directions of silicon, all on the same die.

While $<100>$ and $<110>$ SiBARs show linear TCFs of -10 and $-17 \mathrm{ppm} /{ }^{\circ} \mathrm{C}$ respectively, the phonon trap shows a quadratic temperature characteristic with a turn-over temperature of $87^{\circ} \mathrm{C}$. This is the highest turn-over temperature reported to date for a single crystal silicon resonator realized without the need for ultralow resistivity substrate. Exceeding the industrial temperature range for frequency references, an oven-controlled MEMS oscillator can be implemented by ovenizing this device at the temperature-insensitive turn-over point. Such ovenization, if only provides a temperature control of $\pm 0.1^{\circ} \mathrm{C}$, will be sufficient for realization of references with sub-ppb instability.

\section{SELF-OVENIZED MEMS OSCILLATOR}

An oven-controlled MEMS oscillator is implemented using the $77 \mathrm{MHz}$ phonon trap. Figure 6 shows the frequency behavior of the oscillator for different self-ovenizing DC currents passing through the device body.

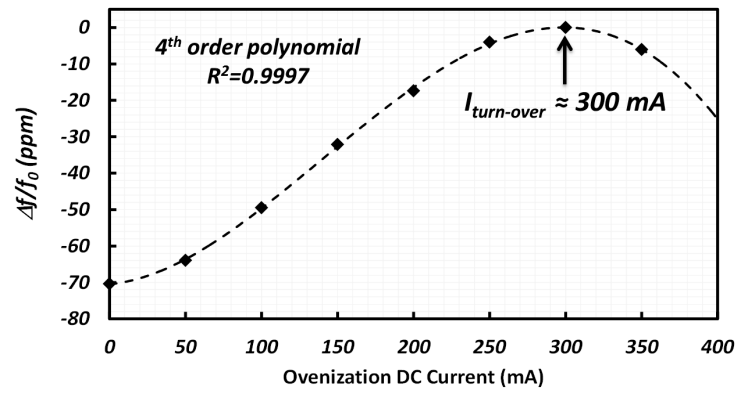

Figure 6: Frequency behavior of oscillator for different selfovenizing DC currents. Ovenization currents can be significantly reduced by proper die layout and vacuum-encapsulation.

A consistent phase-noise of $-106 \mathrm{dBc} / \mathrm{Hz}$ at $1 \mathrm{kHz}$ offset from carrier has been measured from the oscillator for different ovenization currents, including the current required to have the device operating at turn-over point in room-temperature (Figure 7). Such performance demonstrates the potential of the device for realization of highly-stable oven-controlled MEMS oscillators.

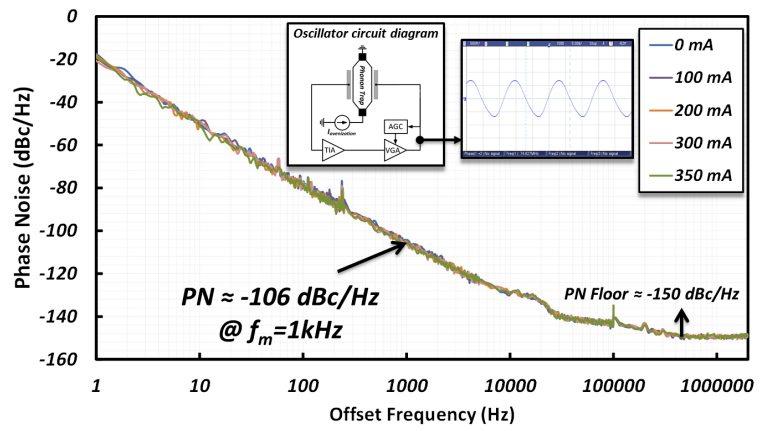

Figure 7: Phase-noise performance of the oscillator for different ovenization DC currents passing through the device body.

\section{REFERENCES}

[1] H. Bhugra, et al, "Commercialization of World's First PiezoMEMS Resonators for High Performance Timing Applications," IEEE MEMS 2014.

[2] R. Tabrizian, et al, "Temperature-Stable Silicon Oxide (SilOx) Micromechanical Resonators," IEEE TED, 2013.

[3] M. Shahmohammadi, et al, "Turnover Temperature Point in Extensional-Mode Highly Doped Si Microresonators," IEEE TED, 2013.

[4] H. M. Lavasani, et al, "Electronic Temperature Compensation of Lateral Bulk Acoustic Resonator Reference Oscillators using Enhanced Series Tuning Technique," IEEE JSSC, 2012.

[5] R. Tabrizian and F. Ayazi, "Acoustically-Engineered MultiPort AlN-on-Silicon Resonators for Accurate Temperature Sensing," IEEE IEDM 2013.

\section{ACKNOWLEDGEMENT}

This work was supported by Integrated Device Technology (IDT), and in part by DARPA TIMU program through SSC pacific contract \# N66001-11-C-4176.

\section{CONTACT}

*R. Tabrizian, tel: +1-404-259-7322; roozbeh@gatech.edu 